\title{
Nondestructive Probing of Perovskite Silicon Tandem Solar Cells Using Multiwavelength Photoluminescence Mapping
}

\author{
Laura E. Mundt, Friedemann D. Heinz, Steve Albrecht, Markus Mundus, Michael Saliba, Juan Pablo Correa-Baena, \\ Elham H. Anaraki, Lars Korte, Michael Grätzel, Anders Hagfeldt, Bernd Rech, \\ Martin C. Schubert, and Stefan W. Glunz
}

\begin{abstract}
In this paper, spatially resolved photoluminescence (PL) spectroscopy with various excitation wavelengths is presented as a nondestructive and versatile technique providing access to the individual subcells of multijunction solar cells. This method is demonstrated on a state-of-the-art monolithic tandem solar cell composed of a planar perovskite solar cell and a silicon heterojunction solar cell. It is shown that the lateral distribution of inhomogeneities can be attributed unambiguously to the individual cells and be related to the manufacturing process. The approach of depth-selective probing of the silicon bottom cell is verified by comparison to reflection maps and by comparison to measurements of the silicon cell after removing the perovskite top cell. Analyzing
\end{abstract}

Manuscript received February 2, 2017; revised March 8, 2017 and March 22, 2017; accepted March 23, 2017. The work of M. Saliba was supported by the Marie Skłodowska Curie fellowship H2020 under Grant 665667. The work of S. Albrecht was supported by the Federal Ministry of Education and Research within the project "Materialforschung für die Energiewende" under Grant 03SF0540. The work of L. E. Mundt, M. C. Schubert, M. Mundus, S. Albrecht, and L. Korte was supported by the German Federal Ministry for Economic Affairs and Energy within the research cluster "Persist" under Grant 0324037A. (Corresponding author: Laura E. Mundt.)

L. E. Mundt, F. D. Heinz, M. Mundus, and M. C. Schubert are with the Department Solar Cells-Development and Characterization, Fraunhofer Institute for Solar Energy Systems, Freiburg 79110, Germany (e-mail: laura.mundt@ise.fraunhofer.de; friedemann.heinz@ise.fraunhofer.de; markus. mundus@ise.fraunhofer.de; martin.schubert@ise.fraunhofer.de).

S. Albrecht is with the Young Investigator Group Perovskite Tandem Solar Cells, Helmholtz-Zentrum Berlin, Berlin 12489, Germany (e-mail: steve.albrecht@helmholtz-berlin.de).

M. Saliba is with the Group for Molecular Engineering of Functional Materials, Institute of Chemical Sciences and Engineering, École Polytechnique Fédérale de Lausanne, Sion 1951, Switzerland (e-mail: michael.saliba@epfl.ch).

J. P. Correa-Baena, E. H. Anaraki, and A. Hagfeldt are with the Laboratory for Photomolecular Science, École Polytechnique Fédérale de Lausanne, Lausanne CH 1015, Switzerland (e-mail: jpcorrea@mit.edu; elham. halvanianaraki@epfl.ch; anders.hagfeldt@epfl.ch).

L. Korte and B. Rech are with the Institute for Silicon Photovoltaics, Helmholtz-Zentrum Berlin, Berlin 12489, Germany (e-mail: korte@ helmholtzberlin.de; bernd.rech@helmholtz-berlin.de).

M. Grätzel is with the Laboratory for Photonics Swiss Federal Institute of Technology, École Polytechnique Fédérale de Lausanne, Lausanne CH 1015, Switzerland (e-mail: michael.graetzel@epfl.ch).

S. W. Glunz is with the Department Solar Cells - Development and Characterization, Fraunhofer Institute for Solar Energy Systems, Freiburg 79110, Germany, and also with the Laboratory for Photovoltaic Energy Conversion, Albert-Ludwigs-University Freiburg, Freiburg 79110, Germany (e-mail: stefan. glunz@ise.fraunhofer.de).

This paper has supplementary downloadable material available at http://ieeexplore.ieee.org.

Color versions of one or more of the figures in this paper are available online at http://ieeexplore.ieee.org.

Digital Object Identifier 10.1109/JPHOTOV.2017.2688022 subcells integrated into a monolithic tandem solar cell is challenging though crucial in order to identify performance limiting loss mechanisms. This method can be used to improve the study of the mutual influence of adjacent subcells in the fully fabricated device, which has been an unfeasible task up to now.

Index Terms-Perovskite, photoluminescence (PL), silicon, tandem.

\section{INTRODUCTION}

$\mathbf{S}$ ILICON wafer based solar cells are dominating the photovoltaic world market, providing $93 \%$ of the total production in 2015 [1]. After more than 30 years of research, this mature technology can draw on a vast variety of advanced characterization techniques and theoretical models. This supported the development of new cell designs enabling record power conversion efficiencies as well as highly efficient, low cost, and long-term stable modules. With a current record efficiency of $26.3 \%$ [2], the single-junction silicon solar cell is approaching the efficiency limit of $29.4 \%$, which is due to Auger recombination [3].

This limitation can be overcome by reducing thermalization losses which can be achieved in a multijunction structure with silicon as bottom cell. Efforts to combine silicon with III-V semiconductors have already been successful using direct wafer bonding to connect the GaInP/AlGaAs top cells with the silicon bottom cell [4]. Since the III-V materials, as well as the involved processes, are rather expensive, an alternative top cell is favored for industrial applications where the main aim is to increase the efficiency without significantly increasing the costs. Organicinorganic halide perovskite solar cells (PSCs) are an emerging technology and have made a remarkable and swift progress in cell efficiency, recently reaching a certified $22.1 \%$ [5]. The continuous progress in increasing the stability of the cells [6]-[10] is also a very important step in becoming a relevant technology for commercial application. PSCs excel due to a sharp optical absorption onset at the band edge, a conveniently tunable band gap in the range of 1.17-2.3 eV [11]-[14], as well as low production costs (due to facile deposition techniques). These beneficial properties suggest combining the advantages of the evolving perovskite-based cells with the already mature silicon technology to form a multijunction solar cell, hence overcoming the efficiency limits of the respective single-junction cells [15], [16]. 
There are two approaches in the development of tandem devices: The cells can be 1) mechanically stacked into fourterminal or 2) series-connected into two-terminal devices. The main advantage of the first approach 1) is that losses due to current mismatch can be avoided giving a larger window of operation at high level. In this four-terminal architecture separate contacts are attached to the top and the bottom cells. Therefore, it is crucial to aim for semitransparent contacts to minimize absorption losses. The development of such tandems cells [17][20] has shown a significant increase of efficiency reaching $25.2 \%$ [21] with the improvement of semitransparent PSCs [6], [22], [23].

Connecting the cells in series 2) with two contacts only implies that the subcell with lower current limits the overall current in the tandem device. Hence, in order to avoid current losses, the band gap (and the thicknesses) of the top and bottom cells has to be carefully matched in a way that the currents of the two subcells coincide under operating conditions. In the case of silicon as a bottom cell with a band gap of $1.12, \mathrm{eV}$ the ideal band gap of a top cell is between 1.6 and $1.9 \mathrm{eV}$ [13], [24], [25]. Although this two-terminal cell design requires an intermediate layer that allows for electrical coupling of the subcells, photon absorption in the back electrode of the top cell and the front electrode of the bottom cell can be minimized. The two-terminal approach is preferred by industry due to the simpler manufacturing process. Recently, successful implementations of these monolithic perovskite silicon tandems were demonstrated [26]-[28] with a certified record efficiency of $23.6 \%$ [29].

The progress in the development of monolithic perovskite silicon tandem solar cells requires characterization techniques that can identify cell efficiency limiting defects and attribute them to the related subcell. One of the most established and versatile methods in the characterization of semiconductors is based on photoluminescence (PL). In the case of silicon wafers and solar cells, PL measurements are used to quantify charge carrier lifetime [30] and surface recombination [31], determination of concentration [32] and type [33] of metal impurities, and other, wafer-based, measurements that can even be used to predict cell efficiencies [34]. In addition, for the characterization of PSCs, PL-based methods are widely used. While local time and spectrally resolved PL measurements are more widely reported [35]-[39], there is also an increasing number of spatially resolved studies [40]-[44].

In this work, we investigate the PL properties of both the top and the bottom cells in a monolithic tandem device in order to evaluate the respective material qualities and to identify possible loss mechanisms. Using different excitation wavelengths, we generate charge carriers in either the top or the bottom cell. The spectrally resolved detection of the PL signal additionally ensures the unambiguous separation of the two cells and allows for precise determination of the perovskite band gap. By analyzing the subcells incorporated in the monolithic device, it is possible to determine the influence of the processing steps of the perovskite cell on the silicon bottom cell, as well as the film quality of the perovskite when processed on a silicon substrate rather than transparent conductive oxide (TCO) glass. The change of the substrate, e.g., involves an effective change in temperature

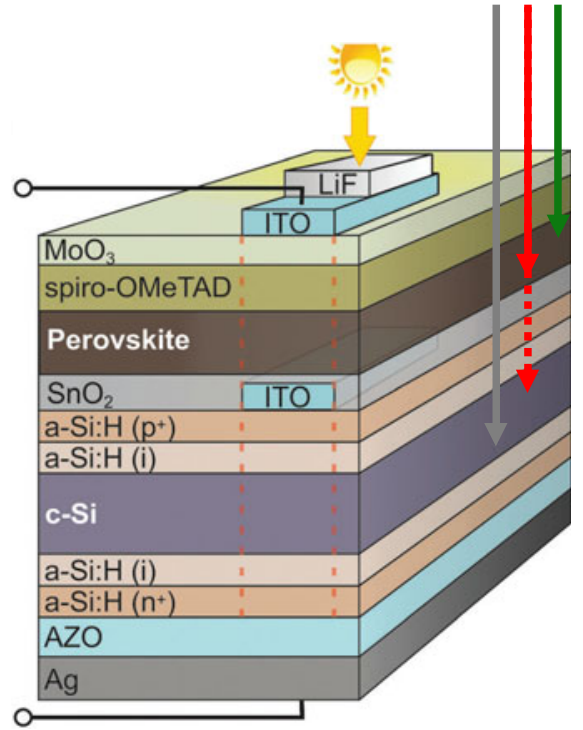

Fig. 1. Schematic structure of a 2-terminal tandem device (reproduced from [4] with permission from The Royal Society of Chemistry) showing the penetration depths of the applied lasers.

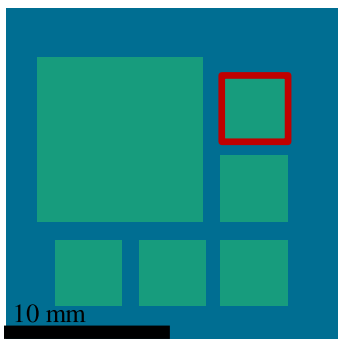

Fig. 2. Schematic overview of cell arrangement on the silicon substrate.

during the process due to different thermal conductivity. With this, we provide an important and easy-to-use tool to accompany the growing field of perovskite/silicon tandem research.

\section{METHOD AND SAMPLE}

The new analytics is demonstrated using a monolithic tandem structure with a planar, Cesium-containing, triple cation perovskite top cell [9] and a silicon heterojunction bottom cell as an example. All process-related details are described in our previous work [28]; the schematic cell structure is depicted in Fig. 1. The silicon substrate $\left(25 \times 25 \mathrm{~mm}^{2}\right)$ contains multiple, electrically isolated tandem solar cells: One with a large active area of $10 \times 10 \mathrm{~mm}^{2}$ and five smaller cells, each with an active area of $4 \times 4 \mathrm{~mm}^{2}$. The analysis of one exemplary cell will be presented in this work, the analysis of a second cell is provided in the Supplementary material. In Fig. 2, the arrangement of the cells is displayed, highlighting the analyzed area. The corresponding cell parameters of the tandem device are given in Table I, the parameters of the second analyzed cell are given in the Supplementary material (see Table I).

All PL measurements were conducted using a micro-PL spectroscopy ( $\mu$-PLS) setup described in [45]. The basis of this setup is a confocal microscope and a moveable XY stage. Different lasers are connected to the microscope via fiber 
TABLE I

Overview of THE CELl PARAMETERS FOR THE ANALYZED CELL

\begin{tabular}{cccc}
\hline \hline$V_{\mathrm{oc}}[\mathrm{V}]$ & $J_{\mathrm{sc}}\left[\mathrm{mA} / \mathrm{cm}^{2}\right]$ & $\mathrm{FF}[\%]$ & $\eta[\%]$ \\
\hline 1.77 & 12.26 & 63 & 13.86 \\
\hline \hline
\end{tabular}

optics and focused onto the sample by a lens. The emitted PL is detected via a fiber coupled monochromator and either a silicon or an InGaAs detector. By scanning the sample the PL spectrum is obtained for every point; the highest achievable resolution is limited by diffraction. The excitation intensity is $7.03 \mathrm{~W} / \mathrm{m}^{2}$ for the $905 \mathrm{~nm}$ laser, $0.11 \mathrm{~W} / \mathrm{m}^{2}$ for the $640 \mathrm{~nm}$ laser, and $0.13 \mathrm{~W} / \mathrm{m}^{2}$ for the $532 \mathrm{~nm}$ laser. Due to the low PL efficiency, a significantly higher excitation intensity has to be used for the silicon PL measurement.

The penetration depth of light into the tandem cell structure depends on its wavelength: while the $532 \mathrm{~nm}$ laser probes the perovskite cell closer to the surface, the red laser penetrates deeper into the material and can also generate charge carriers in the silicon bottom cell (depending on the absorption properties and thicknesses of the overlying layers). The perovskite top cell is almost completely transparent for the infrared laser, except for parasitic absorption especially in the hole transport layer consisting of spiro-OMETAD [26]. Thus, the main charge carrier generation under IR excitation occurs in the silicon bottom cell. A schematic optical path of the different laser beams is shown in Fig. 1. The reflected light as well as the PL is collected by the same lens that is used for focusing the laser beam and passes by a pinhole, only admitting the photons originating from the focus plane to pass and thereby increasing the spatial resolution up to the diffraction limit. Laser filters are applied for the different excitation lasers, in order to attenuate residual laser light on the detector. For the detection of the PL signal emitted from the perovskite and the silicon cell, two different detectors are used. The visible range is detected by a silicon charge-coupled device (CCD) and an InGaAs CCD is used for the near infrared range. Spectrometers with variable gratings offer a very high spectral resolution. By moving the stage including the sample, different areas of interest can be scanned with varying resolution. For every measurement spot, the complete PL spectrum from either 690 to $825 \mathrm{~nm}$ or 840 to $1350 \mathrm{~nm}$, depending on the appropriate detector, is obtained. This allows not only to separately excite the two subcells of the tandem device, but ensures the unambiguous distinction of the PL signals. Fitting the perovskite PL peak by a Gaussian function also allows for the spatially resolved analysis of spectral properties similar to the peak position and therefore the determination of the band gap energy at each position across the device area.

\section{RESULTS}

\section{A. Photoluminescence Spectroscopy}

In Fig. 3, the lateral distribution of the perovskite PL peak wavelength, determined from the $532 \mathrm{~nm}$ measurement, is shown. The map reveals a rather homogeneously distributed band gap energy across the active area.
PSC peak position $532 \mathrm{~nm}$

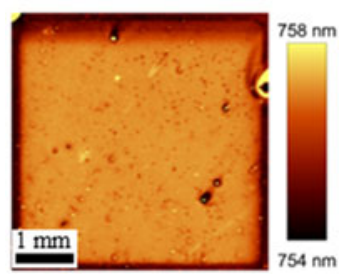

Fig. 3. Lateral distribution of the perovskite PL spectral peak position.

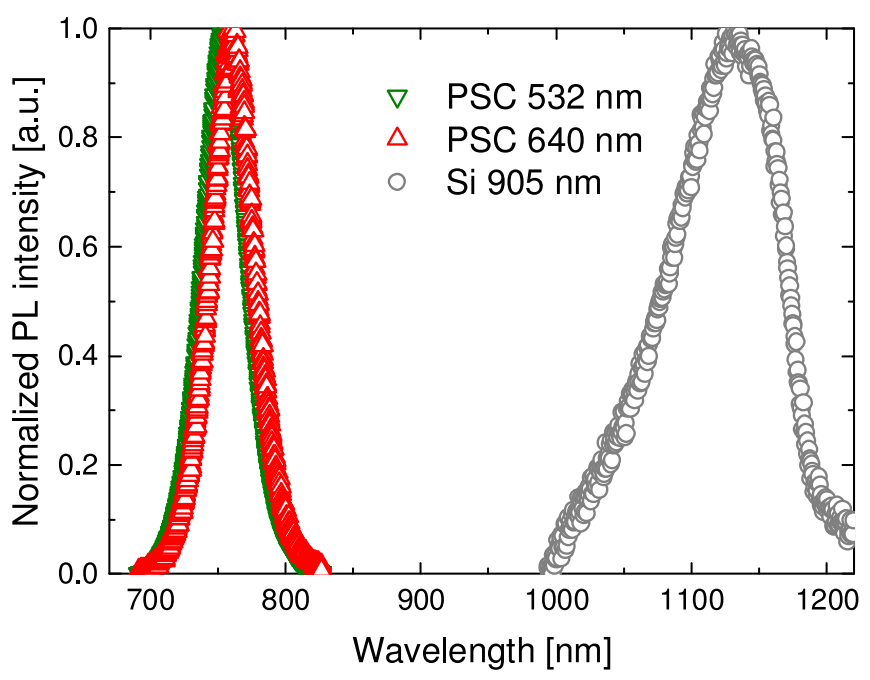

Fig. 4. Normalized photoluminescence spectra measured on a tandem device using three different excitation wavelengths. The spectra are comparable to luminescence spectra in the literature of isolated perovskite or silicon samples.

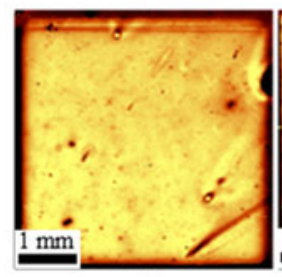

(a)

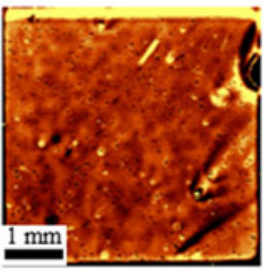

(b)

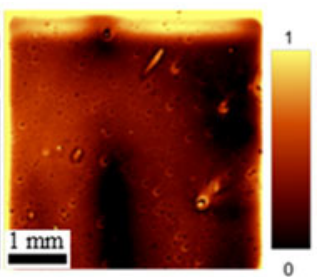

(c)
Fig. 5. Normalized photoluminescence intensity maps for three different excitation wavelengths. Images (a) and (b) show the perovskite cell, while the image (c) corresponds to the silicon cell. (a) PSC $532 \mathrm{~nm}$. (b) PSC $640 \mathrm{~nm}$. (c) $\mathrm{Si}$ $905 \mathrm{~nm}$.

The averaged perovskite PL peak for this triple cation based perovskite is centered at $757 \mathrm{~nm}$ [9], corresponding to a peak-PL derived band gap of $1.64 \mathrm{eV}$.

Note that this is very close to the ideal top cell for tandem applications when using silicon bottom cells [25]. Example PL spectra are depicted in Fig. 4. For better comparability, the spectra have been normalized to their individual maxima.

\section{B. Spatially Resolved Photoluminescence Intensity}

In this section, we present the spatially resolved results: Fig. 5 shows the measured PL intensity maps; comet-like stripes are 


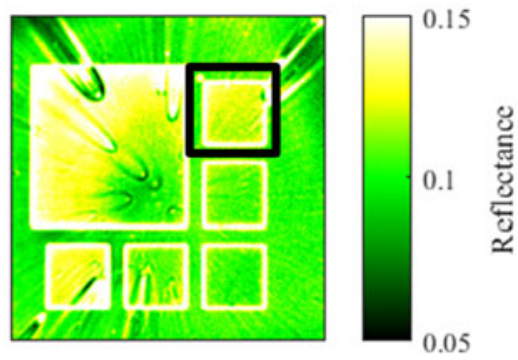

Fig. 6. Reflectance map of the complete substrate for $532 \mathrm{~nm}$, measured with an integrating sphere.

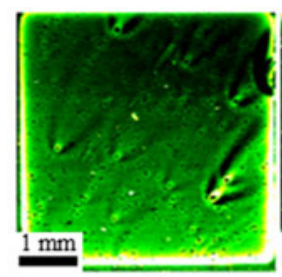

(a)

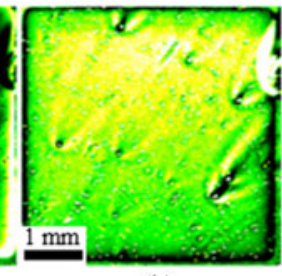

(b)

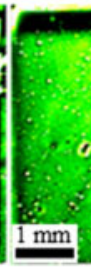

$\mathrm{mm}$
Fig. 7. High resolution confocal reflectance maps for the applied excitation wavelengths, normalized values. (a) $532 \mathrm{~nm}$. (b) $640 \mathrm{~nm}$. (c) $905 \mathrm{~nm}$.

visible in the perovskite PL maps (a-b), especially on the right side of the cell, originating from the center of the silicon substrate containing the cells. The whole pattern is more clearly visible in the reflectance map of the complete substrate in Fig. 6. Two distinct dark spots appear in the lower left region above the scale both in the $532 \mathrm{~nm}$ and in the $640 \mathrm{~nm}$ measurement which correspond to layer damage due to contacting the top ITO layer with metal pins in order to perform electrical measurements.

The maps measured with the $640 \mathrm{~nm}$ laser in Fig. 5(b) exhibit additional interesting substructures: first small dark spots with extensions of 10-30 $\mu \mathrm{m}$ can be observed and second, a distinct mottled pattern is visible all over the cells in the range of hundreds of microns.

The PL map of the silicon cell measured with the $905 \mathrm{~nm}$ laser excitation in Fig. 5(c) displays the point-like spots as well as some stripes. Additionally, larger areas with lower PL intensities can be observed in the silicon cell.

\section{Reflectance Maps}

The absolute reflectance was measured for the complete substrate and several wavelengths using an integrating sphere. Exemplarily the reflectance map for $532 \mathrm{~nm}$ is shown in Fig. 6. The map clearly shows a radially symmetric pattern originating from the substrate center caused by the spin-coating processing for PSCs and a higher reflection for the analyzed cell than for the others that could cause the lower short-circuit current (see Table S1 in the suplementary material). The highest resolution for this method is $25 \mu \mathrm{m} / \mathrm{pixel}$, thus much lower than the resolution of the $\mu \mathrm{PL}$ map. In order to obtain a higher spatial resolution, the confocal setup was used to record the reflected excitation light at 532, 640, and $905 \mathrm{~nm}$ wavelength (see Fig. 7). Special attention has to be paid concerning the origin of the reflected light. Compared to a measurement of the PL emission,

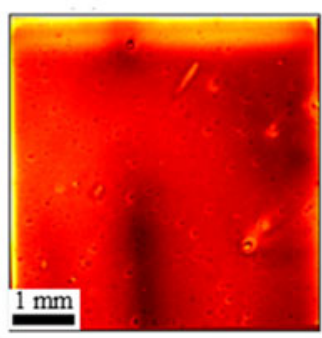

(a)

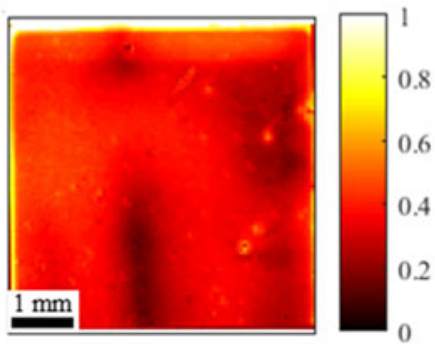

(b)
Fig. 8. Original (a) and reflectance compensated (b) PL map for the silicon bottom cell A. (a) PL 905 nm. (b) Reflectance compensated PL.

all interfaces and intermediate layers in the range of the penetration depth contribute to the measurable reflectance signal. Neither the sponge-like pattern nor the large dark features appear in the 640 or the $905 \mathrm{~nm}$ map, respectively.

\section{DISCUSSION}

The radially symmetric patterns in the PL maps can be attributed to process-related inhomogeneities. The presumed variation in the layer thickness is most likely caused by particles/agglomerates on the substrate impairing the spin coating process and therefore causing an unevenly deposited layer with comet-like features. This effect has been observed before [40]. The manufacturing process of the analyzed device includes two spin-coating steps, depositing the triple cation perovskite in the one-step approach using solvent quenching [9], [28] and the hole transport layer spiro-OMETAD. The identification of the point-like structure requires more statistical sampling over many batches.

The mottled pattern in the $640 \mathrm{~nm}$ map in Fig. 5(b) might be caused by either interference due to an inhomogeneously deposited perovskite layer or varying surface recombination. Since the $640 \mathrm{~nm}$ laser penetrates the material deeper than the shorter wavelength laser and they do not appear in any of the reflectance maps, it can be assumed that these larger substructures originate from within the perovskite layer.

The micrometer-sized features in the $905 \mathrm{~nm}$ maps in Fig. 5(c) that have also been observed in the perovskite PL maps appear to originate from the perovskite top cell. In order to analyze the origin of the unexpected features that are untypical for silicon PL maps, the spatially resolved reflectance for several wavelengths was measured. The observed pattern in the $905 \mathrm{~nm}$ reflectance maps support the hypothesis that these features are optical effects and do not originate in the silicon crystal volume. Thus, a compensation of the reflectance artifacts in the $905 \mathrm{~nm}$ PL map may be done: A higher reflectance means a lower excitation density in silicon. To first order the PL intensity is linearly proportional to the injection density at moderate injection conditions. Fig. 8(b) shows the reflectance-compensated PL map $\mathrm{PL}^{\prime}=\mathrm{PL} /(1-A \times R)$, both maps $(\mathrm{PL}, R)$ were obtained with the confocal setup. A scaling factor $A$ is introduced since the reflectance map is not calibrated to absolute values. It can be seen that different features in the PL image Fig. 8(a) (e.g., 
$\mathrm{Si}$

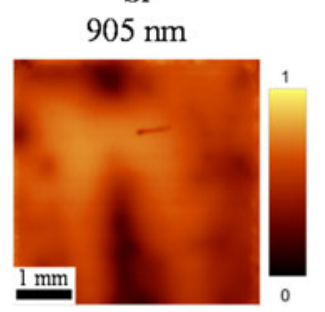

Fig. 9. Normalized PL map of the silicon bottom cell after the removal of the perovskite top cell.

bright area at the top as well as different localized spots) are attenuated and can thus be attributed to optical effects.

In order to cross-check our interpretation of the $905 \mathrm{~nm}$ excitation PL maps, we removed the perovskite top cell including the top ITO layer using ethanol and hydrochloric acid and repeated the PL measurement on the silicon bottom cell. The corresponding PL map with $905 \mathrm{~nm}$ excitation does not display any microfeature (see Fig. 9). This confirms the hypothesis that the microfeatures, which also appeared in the reflectance maps before etching, are optical effects in the top cell. The bare silicon cells exhibit the same larger area features with lower PL intensity that were also apparent in the measurement of the silicon cells still incorporated in the complete tandem device. Such extended defects are common in silicon, as several detrimental impurities in silicon feature a macroscopic diffusion length (e.g., $\mathrm{Cu}$ at room temperature or Fe at elevated processing temperatures [33]).

\section{CONCLUSION}

In this work, we demonstrated a technique to separately analyze both subcells in a monolithic perovskite silicon tandem solar cell in a nondestructive way. Using variable laser excitation wavelength, we could probe the PL emission and light reflection in different layers with micrometer resolution. In particular, we are able to access the silicon bottom cell included in the device by using infrared excitation light for which the perovskite top cell is transparent. Optical effects in the silicon PL map could be distinguished from extended silicon bulk defects by comparison to reflectance maps. This technique yields a unique approach to characterize monolithic perovskite silicon tandem cells in different stages of processing, and hence the influence of succeeding processing steps on the silicon base materials, therefore excluding discrepancies due to film forming variations on different substrates when changing from TCO glass to silicon substrates. We provide a nondestructive, straightforward method to characterize silicon perovskite tandems layer by layer and thus provide valuable feedback throughout the processing.

\section{REFERENCES}

[1] Fraunhofer ISE, Photovoltaics Report, Updated: Jun. 62016.

[2] HERCULES Workshop 2016.

[3] A. Richter, M. Hermle, and S. W. Glunz, "Reassessment of the limiting efficiency for crystalline silicon solar cells," IEEE J. Photovolt., vol. 3, no. 4, pp. 1184-1191, Oct. 2013.
[4] R. Cariou et al., "Monolithic two-terminal III.V//Si triple-junction solar cells With $30.2 \%$ efficiency under 1-Sun AM1.5g," IEEE J. Photovolt., vol. 7, no. 1, pp. 367-373, Jan. 2017.

[5] M. A. Green, K. Emery, Y. Hishikawa, W. Warta, and E. D. Dunlop, "Solar cell efficiency tables (version 48)," Prog. Photovolt., vol. 24, no. 7, pp. 905-913, 2016.

[6] K. A. Bush et al., "Thermal and environmental stability of semitransparent perovskite solar cells for tandems enabled by a solutionprocessed nanoparticle buffer layer and sputtered ITO electrode," Adv. Mater, vol. 28, no. 20, pp. 3937-3943, 2016.

[7] W. Li et al., "Enhanced UV-light stability of planar heterojunction perovskite solar cells with caesium bromide interface modification," Energy Environ. Sci., vol. 9, no. 2, pp. 490-498, 2016.

[8] J. You et al., "Improved air stability of perovskite solar cells via solutionprocessed metal oxide transport layers," Nature Nanotechnol., vol. 11, no. 1, pp. $75-81,2016$.

[9] M. Saliba et al., "Cesium-containing triple cation perovskite solar cells: Improved stability, reproducibility and high efficiency," Energy Environ. Sci., vol. 9, no. 6, pp. 1989-1997, 2016.

[10] M. Saliba et al., "Incorporation of rubidium cations into perovskite solar cells improves photovoltaic performance," Science, vol. 354, no. 6309, pp. 206-209, 2016.

[11] G. E. Eperon et al., "Formamidinium lead trihalide: A broadly tunable perovskite for efficient planar heterojunction solar cells," Energy Environ. Sci., vol. 7, no. 3, pp. 982-988, 2014.

[12] J. H. Noh, S. H. Im, J. H. Heo, T. N. Mandal, and S. I. Seok, "Chemical management for colorful, efficient, and stable inorganic-organic hybrid nanostructured solar cells," Nano Lett., vol. 13, no. 4, pp. 1764-1769, 2013.

[13] M. Anaya et al., "Optical analysis of $\mathrm{CH} 3 \mathrm{NH} 3 \mathrm{SnxPb} 1-\mathrm{xI} 3$ absorbers: A roadmap for perovskite-on-perovskite tandem solar cells," J. Mater Chem. A, vol. 4, no. 29, pp. 11214-11221, 2016.

[14] F. Hao, C. C. Stoumpos, R. P. H. Chang, and M. G. Kanatzidis, "Anomalous band gap behavior in mixed $\mathrm{Sn}$ and $\mathrm{Pb}$ perovskites enables broadening of absorption spectrum in solar cells," J. Amer. Chem. Soc., vol. 136, no. 22, pp. 8094-8099, 2014.

[15] T. P. White, N. N. Lal, and K. R. Catchpole, "Tandem solar cells based on high-efficiency c-Si bottom cells: Top cell requirements for $>30 \%$ efficiency," IEEE J. Photovolt., vol. 4, no. 1, pp. 208-214, Jan. 2014.

[16] M. Filipič et al., "CH3NH3PbI3 perovskite / silicon tandem solar cells: characterization based optical simulations," Opt. Express, vol. 23, no. 7, pp. A263-A278, 2015.

[17] P. Löper et al., "Organic-inorganic halide perovskite/crystalline silicon four-terminal tandem solar cells," Phys. Chem. Chem. Phys., vol. 17, no. 3, pp. 1619-1629, 2015.

[18] D. P. McMeekin et al., "A mixed-cation lead mixed-halide perovskite absorber for tandem solar cells," Science, vol. 351, no. 6269, pp. 151155, 2016.

[19] T. Duong et al., "Semitransparent perovskite solar cell with sputtered front and rear electrodes for a four-terminal tandem," IEEE J. Photovolt., vol. 6, no. 3, pp. 679-687, May 2016.

[20] F. Lang et al., "Perovskite solar cells with large-area CVD-graphene for tandem solar cells," J. Phys. Chem. Lett., vol. 6, no. 14, pp. 2745-2750, 2015.

[21] J. Werner et al., "Efficient near-infrared-transparent perovskite solar cells enabling direct comparison of 4-terminal and monolithic perovskite/silicon tandem cells," ACS Energy Lett., vol. 1, pp. 474-480, 2016.

[22] C. D. Bailie et al., "Semi-transparent perovskite solar cells for tandems with silicon and CIGS," Energy Environ. Sci., vol. 8, pp. 956-963, 2015.

[23] J. Werner et al., "Sputtered rear electrode with broadband transparency for perovskite solar cells," Sol. Energy Mater. Sol. Cells, vol. 141, pp. 407$413,2015$.

[24] F. Meillaud, A. Shah, C. Droz, E. Vallat-Sauvain, and C. Miazza, "Efficiency limits for single-junction and tandem solar cells," Sol. Energy Mater. Sol. Cells, vol. 90, no. 18-19, pp. 2952-2959, 2006.

[25] S. Albrecht et al., "Towards optical optimization of planar monolithic perovskite/silicon-heterojunction tandem solar cells," J. Opt., vol. 18, no. 6, 2016, Art. no. 64012.

[26] J. P. Mailoa et al., "A 2-terminal perovskite/silicon multijunction solar cell enabled by a silicon tunnel junction," Appl. Phys. Lett., vol. 106, no. 12, 2015, Art. no. 121105.

[27] J. Werner et al., "Efficient monolithic perovskite/silicon tandem solar cell with cell area $1 \mathrm{~cm}(2), " J$. Phys. Chem. Lett., vol. 7, no. 1, pp. 161-166, 2016. 
[28] S. Albrecht et al., "Monolithic perovskite/silicon-heterojunction tandem solar cells processed at low temperature," Energy Environ. Sci., vol. 9, no. 1, pp. 81-88, 2016.

[29] K. A. Bush et al., "23.6\%-efficient monolithic perovskite/ silicon tandem solar cells with improved stability," Nature Energy, vol. 2, 2017, Art. no. 17009 .

[30] J. A. Giesecke, B. Michl, F. Schindler, M. C. Schubert, and W. Warta, "Spatially resolved carrier lifetime calibrated via quasi-steadystate photoluminescence," Energy Procedia, vol. 8, pp. 64-70, 2011.

[31] B. Michl, J. A. Giesecke, W. Warta, and M. C. Schubert, "Separation of front and backside surface recombination by photoluminescence imaging on both wafer sides," IEEE J. Photovolt., vol. 2, no. 3, pp. 348-351, Jul. 2012.

[32] M. C. Schubert et al., "Imaging of metal impurities in silicon by luminescence spectroscopy and synchrotron techniques," J. Electron. Mater., vol. 39, no. 6, pp. 787-793, 2010 .

[33] L. E. Mundt et al., "Spatially resolved impurity identification via temperature- and injection-dependent photoluminescence imaging," IEEE J. Photovolt., vol. 5, no. 5, pp. 1503-1509, Sep. 2015.

[34] B. Michl et al., "Efficiency limiting bulk recombination in multicrystalline silicon solar cells," Sol. Energy Mater. Sol. Cells, vol. 98, pp. 441-447, 2012.

[35] S. D. Stranks et al., "Electron-hole diffusion lengths exceeding 1 micrometer in an organometal trihalide perovskite absorber," Science, vol. 342, no. 6156 , pp. 341-344, 2013.

[36] S. D. Stranks et al., "Recombination kinetics in organic-inorganic perovskites: Excitons, free charge, and subgap states," Phys. Rev. Appl., vol. 2 , no. 3, 2014, Art. no. 034007.

[37] Y. Yamada and Y. Kanemitsu, "Photoluminescence spectra of perovskite oxide semiconductors," J. Lumin., vol. 133, pp. 30-34, 2013.
[38] Y. Tian et al., "Mechanistic insights into perovskite photoluminescence enhancement: Light curing with oxygen can boost yield thousand fold," Phys. Chem. Chem. Phys., vol. 17, no. 38, pp. 24978-24987, 2015.

[39] M. de Bastiani, V. D’Innocenzo, S. D. Stranks, H. J. Snaith, and A. Petrozza, "Role of the crystallization substrate on the photoluminescence properties of organo-lead mixed halides perovskites," APL Mater., vol. 2, no. 8, pp. 81509-81515, 2014.

[40] S. Mastroianni et al., "Analysing the effect of crystal size and structure in highly efficient $\mathrm{CH} 3 \mathrm{NH} 3 \mathrm{PbI} 3$ perovskite solar cells by spatially resolved photo- and electroluminescence imaging," Nanoscale, vol. 7, no. 46, pp. 19653-19662, 2015.

[41] Z. Hameiri et al., "Photoluminescence and electroluminescence imaging of perovskite solar cells," Prog. Photovolt., vol. 23, no. 12, pp. 1697-1705, 2015.

[42] A. M. Soufiani et al., "Electro- and photoluminescence imaging as fast screening technique of the layer uniformity and device degradation in planar perovskite solar cells," J. Appl. Phys., vol. 120, no. 3, 2016, Art. no. 35702 .

[43] G. El-Hajje et al., "Quantification of spatial inhomogeneity in perovskite solar cells by hyperspectral luminescence imaging," Energy Environ. Sci., vol. 9, no. 7, pp. 2286-2294, 2016.

[44] M. Vrućinić et al., "Local versus long-range diffusion effects of photoexcited states on radiative recombination in organic-inorganic lead halide perovskites," Adv. Sci., vol. 2, no. 9, 2015, Art. no. 1500136.

[45] P. Gundel, F. D. Heinz, M. C. Schubert, J. A. Giesecke, and W. Warta, "Quantitative carrier lifetime measurement with micron resolution," $J$. Appl. Phys., vol. 108, no. 3, 2010, Art. no. 33705.

Authors' photographs and biographies not available at the time of publication. 